\title{
ANÁLISIS PALEOPARASITOLÓGICO DE COPROLITOS HALLADOS EN SITIOS ARQUEOLÓGICOS DE PATAGONIA AUSTRAL: DEFINICIONES Y PERSPECTIVAS
}

\author{
MARTÍN FUGASSA ${ }^{1}$ Y RICARDO GUICHÓN ${ }^{1,2}$
}

\begin{abstract}
RESUMEN
El estudio de los patrones de salud y enfermedad en las poblaciones antiguas plantea la necesidad de desarrollar interacciones entre especialistas de diversas disciplinas. En este contexto, una línea complementaria e independiente a los estudios de Paleopatología Ósea está comenzando a ofrecer sus primeros resultados para Patagonia: la Paleoparasitología. Esta disciplina emplea el examen de sedimentos, tejidos momificados, huesos y coprolitos en sus intentos por descubrir restos parasitarios. Se han conseguido los primeros resultados paleoparasitológicos para Patagonia Austral y se ha definido una estrategia para el tratamiento y conservación de determinados materiales arqueológicos. En la presente comunicación se describe la metodología empleada en el estudio de coprolitos para dicha región y se enfatiza sobre la importancia de su recuperación y conservación para la discusión de cuestiones paleoecológicas.
\end{abstract}

PALABRAS CLAVES: Coprolito, Paleoparasitología, parásitos, Patagonia Austral.

\section{PALEOPARASITOLOGICAL ANALYSIS IN COPROLITE FOUNDING IN ARCHAEOLOGICAL SITES FROM PATAGONIA AUSTRAL: DEFINING AND PERSPECTIVES}

\begin{abstract}
The study of ancient populations' epidemiological profiles requires joint efforts between specialists of diverse disciplines. In this context, paleoparasitological studies has recently begun to offer independent evidence that supplements more traditional bone palaeopathology studies. Palaeoparasitology analyses sediments, mummified tissue, bones and coprolites in order discover evidence of ancient parasite loads. In this paper/Elsewhere (Cite), the first paleoparasitological results for Southern Patagonia are reported, together with a strategy for the treatment and conservation of specific archaeological materials. Here we outline our methods for the study of coprolites and emphasize the need for recovery and conservation of such remains for paleoecological inferences.
\end{abstract}

KEY WORDS: Coprolite, Palaeoparasitology, parasites, Southern Patagonia.

1 Depto. de Biología, Fac. Cs. Ex. y Nat. Univ. Nac. de Mar del Plata y Depto. de Arqueología, Sede Quequén, Fac. de Cs. Soc., Univ. Nac. del Centro. Argentina. mfugassa@mdp.edu.ar

2 Consejo Nacional de Investigaciones Científicas y Técnicas (CONICET). Argentina. 


\section{INTRODUCCIÓN}

El estudio de los patrones de salud y enfermedad en las poblaciones antiguas plantea la necesidad de desarrollar interacciones entre especialistas de diversas disciplinas, como Historia, Antropología, Arqueología, Medicina y Biología. En este contexto, una línea complementaria e independiente a los estudios de Paleopatología Ósea está comenzado a ofrecer sus primeros resultados para Patagonia: la Paleoparasitología.

Lo que puede interpretarse como un programa de investigación en Ecología Evolutiva Humana ha ofrecido un marco de expectativas generales (Guichón 1994, 2000, 2002) para la región austral ${ }^{1}$, surgido de la interacción con otros grupos (Cocilovo y Guichón 1999/2000, Borrero et al. 2001, Guichón et al. 2000, 2001a y 2001b). Específicamente, se han formulado los primeros esquemas interpretativos tendientes a obtener predicciones y explicaciones concernientes a dichos patrones de salud y enfermedad durante el contacto indirecto y el contacto directo en Tierra del Fuego (Casali y Fugassa 2005 y Fugassa y Guichón 2004). Los recientes hallazgos de huevos, quistes y larvas de parásitos tanto en coprolitos como en sedimentos arqueológicos de la región, implican la incorporación de elementos empíricos que abren un panorama impensado hasta hace poco tiempo.

La Paleoparasitología consiste en el examen microscópico de sedimentos, tejidos momificados, huesos y coprolitos. Estos últimos se forman por deshidratación o mineralización de la materia fecal (Bouchet et al. 2003b). El análisis paleoparasitológico puede exponer la presencia de huevos y larvas de helmintos (nematodes, cestodes, trematodes y acantocéfalos), quistes de protozoos (Entamoeba sp., Eimeria sp., Sarcocystis hominis, etc.) y ectoparásitos. Con estas evidencias, junto a las provistas por otras disciplinas, se reconstruyen aspectos culturales tales como los hábitos higiénicos (Faulkner et al. 2000 y Matsui et al. 2003), el uso de plantas medicinales (Miranda Chaves y Reinhard 2003 y Reinhard et al. 1985), los ítems alimentarios (Bouchet et al. 2001 y, Moore y Fry 1969), el uso del espacio (Fernándes et al. 2005,

1 Región de Magallanes (Chile) y Provincias de Tierra del Fuego y Santa Cruz (Argentina).
Jones 1985, Pike 1968 y Rhode 2003); aspectos ecológicos como la asociación con otras especies (Dittmar y Teejen 2003), grados de agregación en las poblaciones (Santoro et al. 2003), dispersión de las enfermedades (Araujo et al. 1988b, Bouchet et al. 2002 y Nozais 2003); y cuestiones evolutivas relacionadas a la simbiosis parásito-hospedador (Araujo y Ferreira 2000 y Loirelle y Bouchet 2003). Cabe señalar que los estudios paleoparasitológicos se extienden tanto a materiales procedentes de humanos como a fauna arqueológica (Araujo et al. 1988a y Bouchet et al. 2003a) y a contextos paleontológicos (Ferreira et al. 1993). El examen microscópico de coprolitos permite, además de identificar restos parasitarios, evaluar la presencia de epidermis vegetales, granos de polen, esporas y ácaros con lo cual poder sostener con mayores herramientas las inferencias sobre dieta, cultura, salud y ambiente.

A nivel mundial, las investigaciones parasitológicas en sitios arqueológicos tienen su primer antecedente en el hallazgo de Schistosoma haematobium en una momia egipcia (Ruffer, 1910). Trabajos importantes, pero aislados, se fueron sumando (Alisson et al. 1974, Callen y Cameron 1960, Moore 1981, Pike 1968 y Taylor 1955, entre otros) hasta que se conformaron grupos de trabajo en Europa y en América, entre los últimos años de la década de 1970 y principios de la década de 1980. La labor de estos grupos ha sido encabezada por Ferreira (Escola Nacional de Saúde Pública e Instituto Oswaldo Cruz, Brasil), Jones (University of Cork, Inglaterra), Herrmann (Institut fur Aanthropologie, Alemania) y Reinhard (University of Nebraska,USA) (Reinhard 1992). Actualmente, otros grupos como el de la Dra. Bouchet, en Francia, han provisto de nuevas técnicas y novedosos hallazgos. Los estudios se han erigido desde dos enfoques: Ferreira en 1979 designa a esta disciplina como Paleoparasitología (Ferreira 1988) mientras que Reinhard la denominó Arqueoparasitología (Reinhard 1990); la diferencia entre uno y otro radicaría en que los primeros centran su interés en cuestiones biológicas mientras que los segundos han enfocado sus investigaciones en aspectos culturales.

Los primeros estudios paleoparasitológicos de Patagonia Austral fueron realizados sobre sedimentos provenientes de un esqueleto hallado en Cabo Vírgenes (Fugassa et al. 2004). En dicho 
trabajo, si bien no se registraron restos de parásitos, se abordó la problemática propia de Patagonia, definiéndose una metodología específica para la región. A partir de entonces y del análisis de nuevas muestras, se están logrando resultados novedosos y trascendentales que permiten iniciar la discusión acerca de la metodología y la teoría paleoparasitológica sobre una base empírica. En la presente comunicación se hace referencia a la técnica que está siendo aplicada para la región y se discuten las dificultades interpretativas como otras relacionadas con el escaso desarrollo que presenta esta línea de investigación en Patagonia.

\section{SOBRE LA METODOLOGÍA}

Debido a que la forma de los coprolitos es de gran importancia para precisar el origen zoológico del mismo (Chame 2003), éstos son cuidadosamente embalados en frascos para evitar que se disgreguen. Dentro de dichos envases, el material es colocado en sobres de aluminio para aislarlo de cualquier resto orgánico, ya que puede requerir ser datado. La manipulación de todo elemento que conlleva un posterior análisis molecular o microbiológico demanda que se extremen las medidas de higiene para impedir una posible contaminación, por lo cual las muestras se guardan en envases estériles.

El primer paso en el estudio de coprolitos es su descripción exhaustiva. Antes de su disección parcial, los coprolitos se miden y fotografían. La descripción del mismo incluye detallar el color, tanto en su superficie como en su interior, la forma, el tamaño y la presencia de restos macroscópicos tales como pelos, quitinas de insectos, semillas, huesos, etc. (Jouy Avantin et al. 2003). Debido a que los procesos tafonómicos suelen alterar o dispersar los restos que estaban contenidos en los coprolitos, es necesario extraer muestras de los sedimentos en los que estaba inmerso el mismo (Bouchet et al. 2003b). Estas muestras también sirven como control para evaluar la presencia de contaminación del sitio con materia fecal de otro origen.

Para el procesamiento paleoparasitológico, en términos generales, no se examina o procesa la totalidad del coprolito sino que se toman pequeños fragmentos de alrededor de medio gramo. Seguidamente, se depositan en tubos de $15 \mathrm{ml}$ y se rehidrata con fosfato trisódico acuoso 0,5\% (Callen y Cameron 1960) durante 3 días a $4^{\circ} \mathrm{C}$. Al finalizar el período de rehidratación se tritura el material y la suspensión se filtra a un cono IMHOFF o a una copa de sedimentación a través de gasas estériles y se deja sedimentar durante algunas horas (Lutz 1919). La gasa retiene los restos macroscópicos, los cuales se guardan en un frasco con solución fisiológica y formol acético 10\% para posteriores estudios de dieta.

Del material sedimentado en los conos o copas se extraen alícuotas de unos 15 microlitros y se colocan junto a una gota de glicerina sobre un portaobjetos. Se coloca el cubreobjetos y se sella. Estos preparados se observan al microscopio óptico con 100 aumentos y los cuerpos hallados se miden y fotografían con 400 aumentos. El resto del sedimento se trasvasa a viales conteniendo formol acético 10\% para evitar la proliferación de hongos.

Así, al finalizar los análisis sobre una muestra quedan diversos materiales que pasan a conformar lo que aquí se ha dado en llamar una coproteca: coprolitos deshidratados, restos macroscópicos rehidratados y guardados en formol acético, sedimento rehidratado y almacenado en viales, fotografías digitales y preparados sellados. Ningún elemento o fracción del coprolito es eliminado. Para el Proyecto Ecología Evolutiva Humana, la sede Quequén de la Universidad Nacional del Centro de la provincia de Buenos Aires es la depositaria de todo este material - que además incluye muestras comparativas actuales (materia fecal actual de zorros, pumas, etc.)- para estar disponible en futuros estudios.

\section{CONSIDERACIONES SOBRE LOS RESULTADOS Y LAS POSIBLES INFERENCIAS}

Si bien los estudios en Patagonia Austral se han iniciado recientemente, los primeros resultados sobre coprolitos han permitido identificar restos parasitarios en todas las muestras analizadas hasta el momento, identificándose al menos catorce especies de parásitos en restos humanos (Fig. 1); incluso algunas de ellas constituyen los primeros registros en América o en el mundo. Los exámenes se han extendido sobre muestras no humanas, tales como coprolitos de zorros, camélidos y felinos, lo que otorga una descripción más amplia de las parasitosis intestinales durante finales del Pleistoceno y el 
Holoceno y de las relaciones ecológicas acontecidas durante dicho período. Por otra parte, el estudio paleoparasitológico sobre muestras de fauna asociada al hombre puede ofrecer mayor diversidad de especies parasitarias ya que el ser humano moderno pudo reducir la ocurrencia de parasitismos mediante pautas culturales tales como la cocción de los alimentos y las prácticas de higiene.

Las muestras de coprolitos son un material que debe ser cuidadosamente manipulado. En general, los coprolitos son embalados en bolsas y éstas apiladas dentro de cajas. Como ya se mencionó, el uso de envases rígidos y estériles es imprescindible.
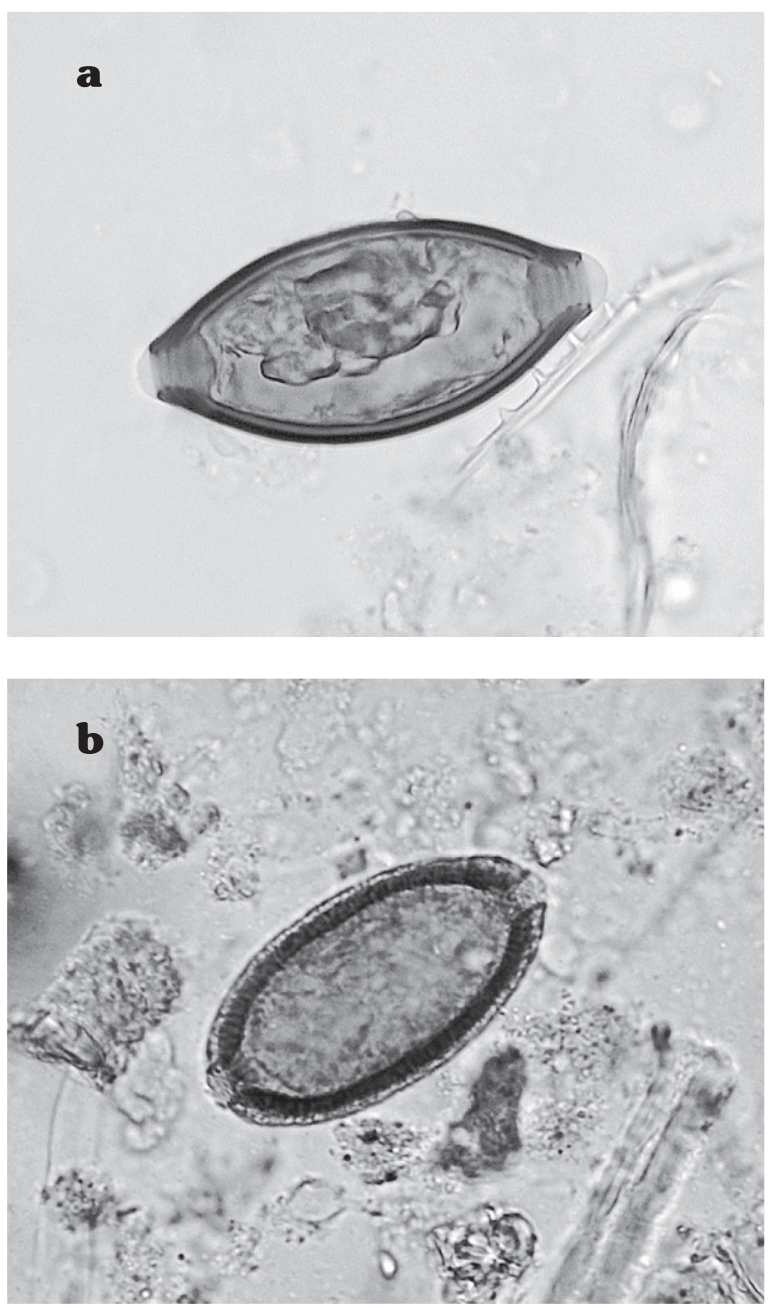

Jouy Avantin et al. (2003) sostienen la importancia de conseguir un método de estandarización en la descripción de coprolitos y propone una ficha con lo cual establecer criterios descriptivos que puedan ser comparables. En este sentido, hay aspectos que podrían tornar subjetiva a la observación; por ejemplo, el color del coprolito es aconsejable que se asigne sobre la base de una escala como la escala Munsell para sedimentos.

La identificación de coprolitos no suele ser fácil y muchas veces sólo el material que conserva rasgos distintivos de materia fecal es rescatado. Frecuentemente, los coprolitos se presentan deformados por diversos motivos como, por ejemplo, el pisoteo mientras que en otras se hallan fragmentados en concreciones menores y poco discernibles. La presencia de restos de parásitos intestinales en dichas concreciones permite establecer si las mismas son de origen intestinal (Jones 1990).

La interpretación de los resultados es otra instancia importante y difícil en los estudios paleoparasitológicos. Una multitud de estructuras microscópicas pueden confundir el diagnóstico y arrojar resultados falsos positivos. Artefactos tales como huevos de nematodes de vida libre (no parásitos) y de ácaros, granos de polen, esporas, estructuras fúngicas y hasta burbujas de aire pueden ser mal diagnosticadas como parásitos y es lo que se conoce como pseudoparasitismo (Thienpont et al. 1979).

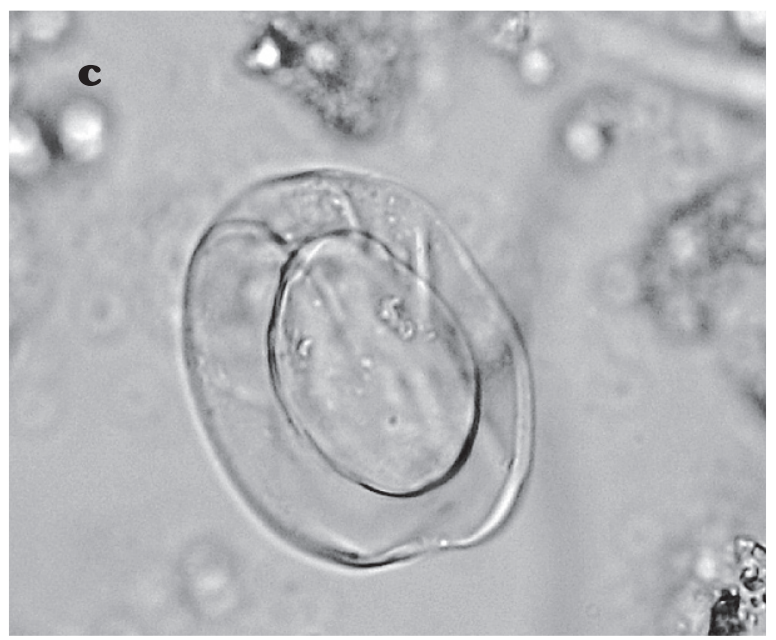

Fig. 1: (a) huevo de Trichuris sp. y (b) huevo de Capillaria sp. en un coprolito humano hallado en CCP5, Parque Nacional Perito Moreno, provincia de Santa Cruz, Argentina (6540 \pm 110 años AP). (c) Huevo de

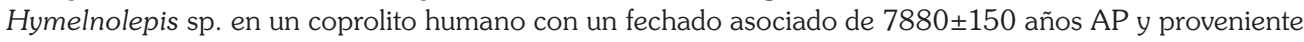
del sitio CCP7 en el Parque Nacional Perito Moreno, provincia de Santa Cruz, Argentina. 
Otra fuente de errores a la hora de establecer inferencias, se suscita por el desconocimiento de los ciclos biológicos de los parásitos. A modo de ilustración, por ejemplo, la presencia de huevos de Capillaria hepatica -nematodes que se alojan en el hígado- en materia fecal de un zorro o un humano no significa que éste estuviese parasitado sino que habría consumido alguna presa -por caso, un ratón- efectivamente parasitada. La presencia de parásitos de las presas en el contenido intestinal de un predador es lo que se conoce como parasitismo en tránsito.

Finalmente, existe la posibilidad de obtener resultados falsos negativos. Estos resultados suceden cuando el parasitólogo que analiza la muestra espera hallar sólo determinadas parasitosis propias de la especie que analiza y rechaza la posibilidad de que puedan encontrarse parásitos poco usuales para determinado hospedador. Por ejemplo, en coprolitos humanos dicho observador esperará encontrar Ascaris lumbricoides, Trichuris trichura y otros específicos de humanos, sin detectar la presencia de otros. Sin embargo, el conocimiento de la diversidad parasitaria y de la plasticidad de muchos parásitos para colonizar especies hospedadoras atípicas puede facilitar el diagnóstico. Es evidente que el marco de expectativas desde el cual se examina una muestra condiciona fuertemente al observador.

Las dificultades que existen en el hallazgo de determinados parásitos en las muestras arqueológicas están siendo superadas, al menos parcialmente, mediante el empleo de técnicas moleculares. Parásitos que se conservan escasamente en sedimentos orgánicos tales como Enterobius vermicularis o aquellos que se hospedan en los tejidos como Trypanosoma cruzi, han sido diagnosticados mediante técnicas de DNA (Iñiguez et al. 2003a, 2003b y Ferreira et al. 2000); similares resultados se han obtenido al aplicar inmunoensayos para identificar protozoos intestinales (Carvahlo Goncalves et al. 2002).

$\mathrm{Al}$ inicio de los estudios paleoparasitológicos en Patagonia Austral surgió como problema la falta de muestras, tanto de coprolitos como de otros sedimentos. La interacción con arqueólogos con larga trayectoria en la región y el diseño de un protocolo para la extracción de sedimentos de esqueletos (Fugassa y Guichón 2005) ha incrementado sustancialmente la existencia de muestras adecuadas sobre las cuales realizar los estudios. Aparentemen- te, la falta de coprolitos es una combinación de la escasez de sitios con buena conservación y de la incomunicación entre arqueólogos y parasitólogos. Esta incomunicación ha retrasado el desarrollo de una disciplina que ha demostrado que puede aportar valiosa información para la reconstrucción de la historia de las poblaciones antiguas. Se espera que los resultados que se están obteniendo para Patagonia Austral fomenten el interés por el tema.

\section{AGRADECIMIENTOS}

A la Dra. María Teresa Civalero (CONICETINAPL) por sus aportes y por confiar en nuestro trabajo. Al Dr. Adauto Araujo (Escola Nacional de Saúde Pública e Instituto Oswaldo Cruz, Brasil), a la Dra. Norma Sardella y al Dr. Guillermo Denegri (Depto. de Biología de la UNMdP) por su permanente apoyo. A nuestras familias. El estudio se realizó como parte del proyecto Ecología Evolutiva Humana en Patagonia (SECYT-UNMDP № 04-09929- 20012002), Ecología Evolutiva Humana en Tierra del Fuego (PICT 2003 № 13889-2005-2007) y el Convenio de Colaboración entre el Instituto Canario de Bioantropología del OAMC de Tenerife, España y la Fac. de Cs. Sociales de la Univ. Nac. del Centro, Argentina (2003-2006).

\section{BIBLIOGRAFÍA}

ALISSON, M.J., PEZZIA, A., HASEGAWA, I. y GERSZTEN, E. 1974. A case of hookworm infection in a pre-Columbian American. American Journal of Physical Anthropology 41: 103-106.

ARAUJO, A., CONFALONIERI, U. y FERREIRA, L.F. 1988a. Oxiurid infestations in small animals from $9000 \mathrm{BP}$ in Brazil. En Paleoparasitologia No Brasil. Ed.PEC-ENSP. Río de Janeiro.

ARAUJO, A., FERREIRA, L.F., CONFALONIERI, U. y CHAME, M. 1988b. Hookworms and the peopling of America. Cadernos de Saúde Pública 2(4): 226-233.

ARAUJO, A. Y FERREIRA, L.F. 2000. Paleoparasitology and the antiquity of human host-parasite relationships. Memorias do Instituto Oswaldo Cruz 95(suppl.I): 89-93.

BORRERO L.A., GUICHÓN, R.A., TYKOT, R., KELLY, J. PRIETO, A. y CÁRDENAS, P. 2001. Dieta a partir de isótopos estables en restos óseos humanos de Patagonia Austral. Estado Actual y perspectivas. Anales del Instituto de la Patagonia, Serie Ciencias Humanas 29: 119-127.

BOUCHET, F., WEST, D., LEFÈVRE, CH. y CORBETT, D. 2001. Identification of parasitosis in a child burial from Adak Island (Central Aleutian Islands, Alaska). Life Science 324: 123-127. 
BOUCHET, F., HARTER, S., PAICHELER, J.C., ARAUJO, A. y FERREIRA, L.F. 2002. First recovery of Schistosoma mansoni eggs from a Latrine in Europa (15-16 ${ }^{\text {th }}$ centuries). The Journal of Parasitology 88(2): 404-405.

BOUCHET, F., ARAUJO, A., HARTER, S., MIRANDA CHAVES, S., DUARTE, A.N., MONNIER, J.L. y FERREIRA, L.F. 2003a. Toxocara canis (Werner, 1782) eggs in the pleistocene site of Menez-Dregan, France (300000500000 years before present). Memorias do Instituto Oswaldo Cruz 98(suppl.I): 137-140.

BOUCHET, F., GIDON, N., DITTMAR, K., HARTER, S., FERREIRA, L.F., MIRANDA CHAVES, S. REINHARD, K.J. y ARAUJO, A. 2003b. Parasite remains in archaeological sites. Memorias do Instituto Oswaldo Cruz 98(suppl.I): 47-52.

CALLEN, E.O. y CAMERON, T.W.M. 1960. A prehistoric diet revealed in coprolites. New Scientist 8:35-40.

CARVAHLO GONCALVES, M.L., ARAUJO, A., DUARTE, R. y PEREIRA DA SILVA, J. 2002. Detection of Giardia duodenalis antigen in coprolites using a commercially available enzyme-linked immunoabsorbent assay. Transactions of Royal Society of Tropical Medicine and Hygiene 96: 640-643.

CASALI, R. y FUGASSA, M.H. 2005. Propuesta metodológica para el estudio de la epidemiología del Contacto europeo-indígena en Tierra del Fuego. Workshop de Historia de la Salud y la Enfermedad. Univ. Nac. de Mar del Plata. Mar del Plata. MS.

CHAME, M. 2003. Terrestrial mamad feces: a morphometric summary and description. Memorias do Instituto Oswaldo Cruz, 98(suppl.I): 95-102.

COCILOVO, J.A. y GUICHÓN, R.A. 1999/2000. La variación geográfica y el proceso de micro-diferenciación de las poblaciones aborígenes de Patagonia Austral y de Tierra del Fuego. Revista Chilena de Antropología 15: 9-28.

DITTMAR, K. y TEEJEN, WR. 2003. The presence of Fasciola hepatica (liver fluke) in human and cattle from a 4500 years old archaeological site in the Saale-Unstrut Valley, Germany. Memorias do Instituto Oswaldo Cruz 98(suppl.I): 141-144.

FAULKNER, CH.T., COWIE, S.E., MARTIN, P.E., MARTIN, S.R., SHANE MAYES, C. y PATTON, SH. 2000. Archaeological evidence of parasitic infection from the $19^{\text {th }}$ century Company Town of Fayette, Michigan. The Journal of Parasitology 86(4): 846-849.

FERNANDES, A., FERREIRA, L.F., CARVAHLO GONCALVES, M.L., BOUCHET, F., KLEIN, C.H., IGUCHI, T., SIANTO, L. y ARAUJO, A. 2005. Intestinal parasite analysis in organic sediments collected from a 16thcentury Belgian archaeological site. Cadernos de Saúde Pública 21(1): 329-332.

FERREIRA, L.F. 1988. A propósito de Paleoparasitología. En Paleoparasitología no Brasil. Ferreira, L.F., Araujo, A. y Confalonieri, U. Eds. PEC/ENSP. Río de Janeiro.

FERREIRA, L.F., ARAUJO, A. y DUARTE, N. 1993. Nematode larvae in fossilized animal coprolites from lower and middle Pleistocene sites, central Italy. The Journal of Parasitology 79(3): 440-442.

FERREIRA, L.F., BRITTO, C., CARDOSO, M.A., FERNANDES, O., REINHARD, K.J. y ARAUJO, A. 2000. Paleopa- rasitology of chagas disease revealed by infected tissue from Chilean mummies. Acta Tropica 75: 79-84.

FUGASSA, M.H. y GUICHÓN, R.A. 2004. Transición epidemiológica en Tierra del Fuego: el contacto indirecto y las enfermedades infecciosas entre 1520 y 1850. Magallania 32: 99-113.

FUGASSA, M.H., GUICHÓN, R.A., DENEGRI, G.M., SARDELLA, N.H y SENATORE, M.X. 2004. Análisis cuantitativo de restos de enteroparásitos en un sitio arqueológico de Patagonia Austral. XV Congreso Nacional de Arqueología Argentina. Río Cuarto, Córdoba. MS.

FUGASSA, M.H. y GUICHÓN, R.A. 2005. Perspectivas para el análisis de sedimentos provenientes de sitios arqueológicos de Patagonia Austral. MS.

GUICHÓN, R.A. 1994. Antropología Física de Tierra del Fuego, caracterización biológica de las poblaciones prehispánicas. Tesis doctoral. Facultad de Filosofía y Letras, Universidad de Buenos Aires. MS.

GUICHÓN, R.A. 2000. Agenda para una Evaluación en la Antropología Biológica de Patagonia Austral. En: Desde el País de los Gigantes, Perspectivas arqueológicas en Patagonia. Ed. UNPA. Río Gallegos.

GUICHÓN, R.A. 2002. Biological Anthropology of Southern Patagonia. En: Archaeological and Anthropological Perspectives on the Native Peoples of Pampa, Patagonia, and Tierra del Fuego to the Nineteenth Century. Claudia Briones, C. y Lanata, J.L. Eds. Greenword. Pub. Co. C.T. USA.

GUICHÓN, R.A., BORRERO, L.A. y MUÑOZ, S. 2000. Datos para una tafonomía de restos humana en Bahía San Sebastián, Tierra del Fuego. Relaciones de la Sociedad Argentina de Antropología 25: 297-311.

GUICHÓN, R.A., BARBERENA, R. y BORRERO, L.A. 2001a. ¿Dónde y cómo aparecen los restos óseos humanos en Patagonia Austral? Anales del Instituto de la Patagonia Serie Ciencias Humanas 29: 103-118.

GUICHÓN, R.A., BORRERO, L.A., PRIETO, A., CÁRDENAS, P. y TYKOT, R. 2001b. Nuevas determinaciones de isótopos estables para Tierra del Fuego. Revista Argentina de Antropología Biológica 3(1): 113-126.

IÑIGUEZ, A.M., ARAUJO, A., FERREIRA, L.F., y VICENTE, A.C.P. 2003a. Analysis of ancient DNA from coprolites: a perspective with random amplified polymorphic DNA-polymerase chain reaction approach. Memorias do Instituto Oswaldo Cruz 98(suppl.I): 63-66.

IÑIGUEZ, A.M., REINHARD, K.J., ARAUJO, A., FERREIRA, L.F., y VICENTE, A.C.P. 2003b. Enterobius vermicularis: ancient DNA from North and South American human coprolites. Memorias do Instituto Oswaldo Cruz 98(suppl.I): 67-70.

JONES, A.K.J. 1985. Trichurid ova in archaeological deposits: their value as indicators of ancient faeces. En Paleobiological investigations: research design, methods and data analysis. Fieller, R.J., Gilbertson, D.D. and Ralph, N.G.A. eds. Symposia of the Association for Environment Archaeological. BAR International Series 266. Heslington.

JONES, A. 1990. Coprolites and fecal concretions. En Brean Down Excavations 1983-1987. London: English Heritage. Archaeological Report 15: 242-245. 
JOUY AVANTIN, F., DEBENATH, A., MOIGNE, A.M. y MONÉ, H. 2003. A standardized method for the description and the study of coprolites. Journal of Archaeological Science 30: 367-372.

LOIRELLE, O. y BOUCHET, F. 2003. Evolution of Ascaris in human and pigs: a multi-disciplinary approach. Memorias do Instituto Oswaldo Cruz 98(suppl.I): 39-46.

LUTZ, A. 1919. Schistosoma mansoni e a schistosomatose segundo observacoes feitas no Brasil. Memorias do Instituto Oswaldo Cruz 11: 121-155.

MATSUI, A., KANEHARA, M. y KANEHARA, M. 2003. Paleoparasitology in Japan. Discovery of toilet features. Memorias do Instituto Oswaldo Cruz 98(suppl. I): $127-136$.

MIRANDA CHAVES, S.A. y REINHARD, K.J. 2003. Paleopharmacology and pollen: theory, meted and application. Memorias do Instituto Oswaldo Cruz 98(suppl. I): 207-211.

MOORE, P.D. 1981. Life seen from a medieval latrine. Nature 294(17): 614.

MOORE, J.G. y FRY, G.F. 1969. Thorny-headed worm infection in North America prehistoric man. Science 163: 1324-1325.

NOZAIS, J.P. 2003. The origin and dispersion of human parasitic diseases in the Old World (Africa, Europe and Madagascar). Memorias do Instituto Oswaldo Cruz 98(suppl.I): 13-20.

PIKE, A.W. 1968. Recovery of helminth eggs from archaeological excavations, and their possible usefulness in providing evidence for the purpose of an occupation Nature 219: 303-304.

REINHARD, K.J. 1990. Archaeoparasitology in North America. American Journal of Physical Anthropology 82: 145-163.

REINHARD, K.J. 1992. Parasitology as an interpretative tool in archaeology. American Antiquity 57(2): 231-245.

REINHARD, K.J., AMBLER, J.A. y McGUFFIE, M. 1985. Diet and parasitism at Dust Devil Cave. American Antiquity 50(4): 819-824.

RHODE, D. 2003. Coprolites from Hidden Cave, revisited: evidence for site occupation history, diet and sex of occupants. Journal of Archaeological Science 30: 909-912.

RUFFER, M.A. 1910. Note on the presence of "Bilharzia haematobia" in Egyptian mummies of the Twentieth Dynasty (1250-1000B.C.). Britannic Medical Journal $1: 16$.

SANTORO, C., DORSEY VINTON, SH. y REINHARD, K.J. 2003. Inca expansion and parasitism in the Lluta Valley: preliminary data. Memorias do Instituto Oswaldo Cruz 98(suppl.I): 161-164.

TAYLOR, E.L. 1955. Parasitic helminthes in medieval remains. Veterinary Record 67: 216-218.

THIENPONT, D., ROCHETTE, F. y VANPARIJS, O.F.J. 1979. Diagnóstico de las helmintiosis por medio del examen coproparasitológico. Janssen Research Foundation. 
